\title{
Cognitive behavioural therapy, systemic behavioural family therapy, and non-directive supportive therapy had similar long term effectiveness for major depressive disorder in adolescents
}

\author{
Birmaher B, Brent DA, Kolko D, et al. Clinical outcome after short-term psychotherapy for adolescents with major \\ depressive disorder. Arch Gen Psychiatry 2000 Jan;57:29-36.
}

QUESTION: In adolescents with major depressive disorder (MDD), is short term psychotherapy effective for relieving symptoms in the long term?

Design

Randomised \{allocation concealed*\}t, blinded (outcome assessor)*, controlled trial with 24 months of follow up.

\section{Setting}

Pittsburgh, Pennsylvania, USA.

Patients

107 adolescents who were $13-18$ years of age (mean age $16 \mathrm{y}, 76 \%$ girls), met DSM-III-R criteria for MDD, and had a Beck Depression Inventory score $\geqslant 13$. Exclusion criteria were ongoing physical or sexual abuse; psychosis; or bipolar, obsessive compulsive, eating, or substance abuse disorders. $92 \%$ of patients completed $\geqslant 7$ of 8 assessments, and $97 \%$ were included in the analyses.

\section{Intervention}

Patients were allocated to 1 of 3 groups: cognitive behavioural therapy (CBT) $(\mathrm{n}=37)$, systemic behavioural family therapy $(\mathrm{n}=35)$, or non-directive supportive therapy $(n=35)$. Psychotherapy lasted 12-16 weeks, and patients were followed up for 24 months thereafter.

\section{Main outcome measures}

Rates of remission ( $\geqslant 2$ weeks and $\leqslant 2$ months with absence of MDD), recovery ( $\geqslant 2$ months with absence of MDD), relapse (an episode of MDD during the period of remission), and recurrence (an episode of MDD during the period of recovery); severity of depression (Kiddie Schedule for Affective Disorders and Schizophrenia and the Beck Depression Inventory); functional status (Children's Global Assessment Scale); cognitive distortion (Children's Negative Cognitive Error Questionnaire and the Beck Hopelessness Scale); family environment (Conflict Behavior Questionnaire, Areas of Change Questionnaire, and Family Assessment Device); and parents' current and lifetime psychiatric disorders (Schedule for Affective Disorders and SchizophreniaLifetime version).

National Institute of

Mental Health.

For correspondence: Dr B Birmaher,

Department of

Psychiatry, University

of Pittsburgh School of

Medicine, Western

Psychiatric Institute

and Clinic, 3811

O'Hara Street,

Pittsburgh, PA 15213

USA.Fax +1412624

3703.

\section{Main results}

The 3 groups did not differ for any of the clinical outcomes at the end of the 2 year follow up. The study had $80 \%$ power to detect effect sizes ranging from $0.30-$ 0.50 . Remission and recovery rates were $84 \%$ and $83 \%$, respectively, for the group as a whole. $30 \%$ of recovered patients had 1 recurrence, and $8 \%$ had 2 recurrences. $21 \%$ of patients continued to have substantial depressive symptoms and poor functioning.

\section{Conclusions}

In adolescents with major depressive disorder, most patients improved after 24 months of follow up, regardless of type of psychotherapy (cognitive behavioural therapy, systemic behavioural family therapy, or nondirective supportive therapy). $30 \%$ of adolescents had recurrences, however, and 21\% had chronic depression.

*See glossary.

$\dagger$ Information provided by author.

\section{COMMENTARY}

Birmaher $e t a$ ls findings of the equivalence of 3 different forms of therapy in adolescents' recovery from depression and recurrence during 2 years of follow up need to be interpreted with caution as to ecological validity. For example, the 12-fold reduction in rate of remission found in the group recruited through advertising raises the question of how different are patients and families who do not respond to advertising but go to clinics for treatment or who seek help in the privacy of traditional, non-institutional, private practice mental health settings. Unfortunately, cautions about the study's ecological validity are likely to be lost in a "keep psychotherapy cheap" climate where some managed healthcare organisations take advantage of the stigma of mental illness and popular prejudices that all therapy is the same. This study should not be used to promote practices such as behavioural carveouts, which limit both resources and choice, or to justify funnelling patients to the cheapest "providers" rather than to the most highly trained clinicians. A subtle but more valid inference from the study's most robust finding is the importance of creating access to mental healthcare systems and practices that promote choice and hope for potential patients. ${ }^{1-3}$

The finding that CBT is effective in the short term but not the long term must be considered cautiously also. In private practice, achieving such meaningful, yet hard to measure, long term mental health outcomes as autonomy and authenticity are more likely to be clinical priorities. ${ }^{4}$ This study confirms clinical experience, which suggests that the keys to effective treatment are attention to the therapeutic alliance and open, non-transference distorted communication rather than simply frequent booster sessions. This is, in effect, an informed consent process rather than the pro forma informed consent of most study designs. ${ }^{4}$

Harold J Bursztajn, MD Harvard Medical School Cambridge, Massachusetts, USA

1 Bursztajn HJ, Brodsky A. Captive patients, captive doctors: clinical dilemmas and interventions in caring for patients in managed health care. Gen Hosp Psychiatry 1999;21:239-48.

2 Meissner WW. The therapeutic alliance. New Haven: Yale University Press, 1996.

3 Illingworth P. Bluffing, puffing and spinning in managed care organizations. J Med Philos 2000;25:62-76.

4 Bursztajn HJ. Efficacy research and psychodynamic psychiatry [letter]. Am J Psychiatry 1991;148:817-18. 